\title{
Characterisation of Sidelobes for Multibeam Radar based on Quasi-Orthogonal LFM Waveforms
}

\author{
Alessio Balleri*, Leon Kocjancic* and Thomas Merlet** \\ ${ }^{*}$ Centre for Electronic Warfare, Information and Cyber, Cranfield University, \\ Defence Academy of the United Kingdom, Shrivenham, SN6 8LA, United Kingdom \\ ${ }^{* *}$ Thales LAS France SAS, Elancourt, 78995, France
}

\begin{abstract}
Multibeam radars (MBRs) enable multiple independent channels by simultaneously exploiting spatial and waveform diversity. Orthogonal waveforms are employed to form multiple independent antenna beams, each one providing a different function and using different dedicated radar resources. This paper investigates sidelobe levels in MBRs and presents a comparison with those of an Electronic Steerable Array (ESA) that employs a single waveform in transmission to generate multiple simultaneous beams. Simulations are carried out for a 3-channel MBR transmitting quasi-orthogonal Linear Frequency Modulated (LFM) waveforms at $\mathrm{Ku}$ band. The response of the MBR to an ideal point target as a function of aspect angle as well as that to multiple targets in different locations has been investigated. Results corroborate the analytical findings and show that the sidelobe levels with respect to angle, at the target range, are attenuated by the cross-ambiguity function properties between the waveforms employed. The range response to a target in low channel isolation suffers from cross-channel interference that may alter the noise floor characteristics of the radar, hence stressing the importance of suitable waveform selection.
\end{abstract}

\section{INTRODUCTION}

Multibeam radars (MBR) use waveform diversity (WD) to form multiple independent beams and provide multiple simultaneous functions in different directions. Each radar channel corresponds to a beam formed by a dedicated waveform that is orthogonal to the waveforms that generate all other beams [1] [2] [3] [4]. To achieve this, each element of the array transmits a linear combination of orthogonal waveforms. On receive, phase steering and waveform orthogonality are employed in conjunction to reduce the mutual interference between adjacent beams and allow MBR to provide multiple simultaneous functions. The main benefits are maximum use of the available bandwidth and increased resource management performance.

The research community has been very active in the area of orthogonal waveform design, particularly to address the need of multiple-input multiple-output (MIMO) radar systems. MIMO radar with collocated antennas is a radar configuration that enables improved parameter estimation, better target detection, beamforming flexibility, improved angular resolution, and minimum detectable velocity [5], [6]. Similarly to MBR, MIMO systems rely on orthogonal waveforms in transmission to provide multiple simultaneous channels all exploiting the entire available bandwidth [7] [8]. The difference is that each element of a MIMO system transmits a waveform that is orthogonal to the waveforms used by all other antenna elements. MIMO radar can be synthesised with collocated or widely separated antennas. When the antennas are far apart, MIMO radar is a type of multistatic radar that exploits spatial diversity of radar cross section (RCS) [9]. Both collocated and multistatic MIMO configurations demand the use of multiple orthogonal waveforms [7]. Numerous approaches have been followed to design orthogonal or pseudoorthogonal waveforms for MIMO radars. Waveforms are often not optimal because radar waveforms can only provide pseudoorthogonality and radar targets are characterised by a timevarying and frequency selective response. A solution proposed in the past is to employ numerically optimised polyphase codes, but these make target detection more difficult because they lack Doppler tolerance [8]. As a result, processing at the receiver becomes more complex due to the requirement of a bank of filters to account for the different Doppler shifts. Doppler tolerance is one of the main advantages of linear frequency modulated (LFM) waveforms [10]. Additionally, when the chirp is not modulated in amplitude, the peak power remains constant and the transmitter can operate under saturation. An up-chirp and down-chirp can be used to form independent beams but this approach is limited to only two beams [1]. Chirp rate diversity can be used to produce more than two channels [3], [11], [12]. In attempting to produce a larger number of orthogonal waveforms, some authors have combined FDM with LFM [13], [14]. However, the existing proposed solutions are based on a numerical optimisation of a matrix of chirps, discretely distributed in time and frequency that result in loss of Doppler tolerance. Chirp rate diversity was also used in telecommunication applications for channel estimation and multiuser channel allocation [15]. Waveforms with a large time-bandwidth (BT) product can be formed by stacking together chirp signals with the same bandwidth to form pseudo-orthogonal waveforms [16]. Although some of the Doppler tolerance is lost, constant amplitude ensures maximum energy in transmission and chirp rate diversity allows for multiple instances of pseudo-orthogonal waveforms. Although no thorough examination to determine optimal parameters of such waveforms has been conducted, a similar approach was followed to numerically optimise chirp rate diversity waveforms [17].

The aim of this paper is to investigate sidelobe levels in MBRs. The solution presented here requires the use of combined signal processing on both transmit and receive. It differs from 
other transmit-only solutions that can provide multiple multifunction beams, without direct use of orthogonal waveforms, such as those presented in [18] and [19]. In this paper, the analytical results on MBR presented in [3] are extended to multiple targets and sidelobe level performance is assessed with a set of simulations for a 3-channel MBR transmitting LFM chirps. A comparison with the case of a typical ESA using only one waveform in transmission to create multiple simultaneous beams is presented.

\section{BACKGROUND THEORY}

Let us consider an antenna array of $K$ omnidirectional elements transmitting signals arranged in a vector

$$
\mathbf{s}(t)=\left[s_{1}(t) s_{2}(t) \cdots s_{K}(t)\right]^{T}
$$

with $s_{k}(t)$ being the transmitted narrowband signal at the $k$ th element of the array. The vector of all echo signals at the receiving elements, reflected from the $\mathrm{i}$-th target in a direction $\theta_{i}$ can be expressed as

$$
\mathbf{y}_{\mathbf{i}}(t)=\gamma_{i} \mathbf{a}\left(\theta_{i}\right) \mathbf{a}^{T}\left(\theta_{i}\right) \mathbf{s}\left(t-t_{i}\right) e^{-j 2 \pi f_{c} t_{i}} e^{j 2 \pi f_{i} t}
$$

where $\gamma_{i} \in \mathbb{C}$ is the complex target reflection coefficient, $t_{i}$ is the echo time-delay, $f_{c}$ is the carrier frequency, $f_{i}$ is the target Doppler shift, and $\mathbf{a}\left(\theta_{i}\right)$ is the manifold $K \times 1$ vector corresponding to the direction $\theta_{i}$ with elements

$$
a_{k}\left(\theta_{i}\right)=e^{j 2 \pi f_{c} d \sin \theta_{i}(k-1) / c}
$$

When $N$ targets are present the vector of all received signals is

$$
\mathbf{y}(t)=\sum_{i=1}^{N} \gamma_{i} \mathbf{a}\left(\theta_{i}\right) \mathbf{a}^{T}\left(\theta_{i}\right) \mathbf{s}\left(t-t_{i}\right) e^{-j 2 \pi f_{c} t_{i}} e^{j 2 \pi f_{i} t}
$$

MBR uses a linear combination of orthogonal waveforms at each antenna element to increase isolation between multiple simultaneous beams that point in different directions [3].

Each antenna element of an MBR transmits a linear combination of $M$ orthogonal waveforms $\mathbf{x}(t)$

$$
\mathbf{s}(t)=\mathbf{W} \mathbf{x}(t)
$$

where $\mathbf{W}$ is a $K \times M$ steering matrix defined as

$$
\mathbf{W}=\left[\mathbf{a}\left(\tilde{\theta}_{1}\right) \mathbf{a}\left(\tilde{\theta}_{2}\right) \cdots \mathbf{a}\left(\tilde{\theta}_{M}\right)\right]^{*}
$$

with the symbol $*$ indicating the conjugate operation. Each column of $\mathbf{W}$ is a steering vector pointing in the direction $\tilde{\theta}_{m}$, for $m=1, \ldots, M$ and the elements of $\mathbf{x}(t)$ are orthogonal waveforms $x_{m}(t)$, each one corresponding to one of the $M$ beams. Inserting Eq. 5 in Eq. 2 leads to the received signal

$$
\mathbf{y}(t)=\sum_{i=1}^{N} \alpha_{i} \mathbf{a}\left(\theta_{i}\right) \mathbf{a}^{T}\left(\theta_{i}\right) \mathbf{W} \mathbf{x}\left(t-t_{i}\right) e^{j 2 \pi f_{i} t}
$$

where $\alpha_{i}=\gamma_{i} e^{-j 2 \pi f_{c} t_{i}}$. Extraction of the $m$ th channel is achieved by matched filtering the received signal at each antenna element to $x_{m}(t)$ and by steering the receiving array in the direction $\tilde{\theta}_{m}$ so to obtain

$$
\begin{aligned}
z_{m}(t) & =\mathbf{a}^{H}\left(\tilde{\theta}_{m}\right)\left[\mathbf{y}(t) \circledast x_{m}^{*}(-t)\right] \\
& =\sum_{i=1}^{N} \alpha_{i} \mathbf{a}^{H}\left(\tilde{\theta}_{m}\right) \mathbf{a}\left(\theta_{i}\right) \mathbf{a}^{T}\left(\theta_{i}\right) \\
& \times\left[\sum_{k=1}^{M} \mathbf{a}^{*}\left(\tilde{\theta}_{k}\right) \chi_{m k}\left(t-t_{i}, f_{i}\right) e^{j 2 \pi f_{i} t}\right]
\end{aligned}
$$

where the symbol $\circledast$ indicates the convolution function, and $\chi_{m k}$ is the cross-ambiguity function between $x_{m}$ and $x_{k}$ defined as

$$
\chi_{m k}\left(\tau, f_{D}\right)=\int_{-\infty}^{\infty} x_{m}^{*}(t) x_{k}(t+\tau) e^{j 2 \pi f_{D} t} d t
$$

Eq. 8 can be rearranged to obtain

$$
\begin{aligned}
z_{m}(t) & =\sum_{i=1}^{N} \beta_{i}(t) \mathbf{a}^{H}\left(\tilde{\theta}_{m}\right) \mathbf{a}\left(\theta_{i}\right) \mathbf{a}^{T}\left(\theta_{i}\right) \mathbf{a}^{*}\left(\tilde{\theta}_{m}\right) \chi_{m m}\left(t-t_{i}, f_{i}\right) \\
& +\sum_{i=1}^{N} \beta_{i}(t) \mathbf{a}^{H}\left(\tilde{\theta}_{m}\right) \mathbf{a}\left(\theta_{i}\right) \mathbf{a}^{T}\left(\theta_{i}\right) \\
& \times\left[\sum_{k \neq m}^{M} \mathbf{a}^{*}\left(\tilde{\theta}_{k}\right) \chi_{m k}\left(t-t_{i}, f_{i}\right)\right]
\end{aligned}
$$

with $\beta_{i}(t)=\alpha_{i}(t) e^{j 2 \pi f_{i} t}$, where the first term represents the co-channel response and the second term represents the contribution of all the adjacent channels. When a set of orthogonal waveforms is employed, $\chi_{m k}\left(\tau, f_{d}\right)=0$ for $k \neq m$ and the contribution of all cross-channels is zero. A detailed mathematical treatment of the signal model for the case of a single target can be found in [3].

The amount of cross-channel interference depends on the isolation between the signals in use and the aspect angle of the target with respect to the direction of each channel antenna beam. For perfectly orthogonal waveforms, the second term in Eq. 10 is null and the response to the target in each channel is the same of that of a typical single beam array. However, in practice, waveforms are only quasi-orthogonal and the crosschannel interference depends on the level of isolation and cross-correlation properties between waveforms as well as the beam pattern associate to all cross-channels in the direction of the target. The difference with a conventional MIMO radar, that requires an orthogonal waveform for each radiating element, is that MBR requires orthogonal waveforms for each independent beam. As a consequence, for MBR, a smaller number of orthogonal waveforms is potentially required and each beam can be associated to waveforms using different resources and providing different performance parameters (e.g. resolution and ambiguities).

The isolation between two signal is defined as

$$
I_{m k}\left(\tau, f_{D}\right)=\left|\frac{\chi_{m m}(0,0)}{\chi_{m k}\left(\tau, f_{D}\right)}\right|=\left|\frac{\chi_{m m}(0,0)}{\chi_{k m}^{*}\left(-\tau,-f_{D}\right)}\right|
$$


Eq. 11 describes the ratio between the amplitude of the cochannel and cross-channel contributions for two independent beams pointing in the same direction. A pair of ideal orthogonal waveforms satisfy the equality $\chi_{m k}\left(\tau, f_{D}\right)=0$ for all values of $\tau$ and $f_{D}$. However, real waveforms will only be pseudo-orthogonal and are commonly designed so that their cross-ambiguity function is lower than a predefined value. Waveforms designed with a high isolation for all possible pairs ensure a small cross-channel interference.

\section{ISOLATION FOR LINEAR CHIRPS}

The isolation properties between waveforms generated by employing linear chirps of the same duration over different bandwidths, to allow for chirp rate diversity, were presented in [3].

Let us consider two baseband chirps $x_{1}(t)$ and $x_{2}(t)$ of the same duration $T$ and of the form

$$
x_{1}(t)=e^{j \pi \gamma_{1} t^{2}} e^{j 2 \pi f_{s 1} t}
$$

and

$$
x_{2}(t)=e^{j \pi \gamma_{2} t^{2}}
$$

for $t \in(-T / 2, T / 2)$ and zero elsewhere. The starting frequency of $x_{1}(t)$ is $f_{s 1}$ and the chirps have bandwidths $B_{1}$ and $B_{2}$, and chirp rates $\gamma_{1}=B_{1} / T$ and $\gamma_{2}=B_{2} / T$, respectively. The bandwidth difference is $\Delta B=B_{2}-B_{1}$ (where the bandwidth is positive for up-chirps and negative for downchirps). The general expressions of the isolation as a function of the waveform design parameters was derived in [3] and for $\tau<0$ can be expressed as

$$
\begin{aligned}
& I_{12}\left(\tau, f_{D}\right)= \\
& \sqrt{2 T \Delta B} \mid F\left(\sqrt{\frac{2 \Delta B}{T}}\left(\frac{T}{2}+\frac{B_{2} \tau-T\left(f_{s 1}+f_{D}\right)}{\Delta B}\right)\right) \\
& -\left.F\left(\sqrt{\frac{2 \Delta B}{T}}\left(-\frac{T}{2}+\frac{B_{1} \tau-T\left(f_{s 1}+f_{D}\right)}{\Delta B}\right)\right)\right|^{-1}
\end{aligned}
$$

and for $\tau>0$

$$
\begin{aligned}
& I_{12}\left(\tau, f_{D}\right)= \\
& \sqrt{2 T \Delta B} \mid F\left(\sqrt{\frac{2 \Delta B}{T}}\left(-\frac{T}{2}+\frac{B_{2} \tau-T\left(f_{s 1}+f_{D}\right)}{\Delta B}\right)\right) \\
& -\left.F\left(\sqrt{\frac{2 \Delta B}{T}}\left(\frac{T}{2}+\frac{B_{1} \tau-T\left(f_{s 1}+f_{D}\right)}{\Delta B}\right)\right)\right|^{-1}
\end{aligned}
$$

where $F(\cdot)$ is the complex form of Fresnel integral. The detailed calculations demonstrating Eq. 14 and Eq. 15, including more general results, can be found in [3]. Eq. 14 and Eq. 15 can be approximated to derive a lower bound on the isolation

$$
\bar{I}_{12}>\sqrt{\frac{\Delta B T}{2}}
$$

Note, that Eq. 16 is a bound and isolation tends to increase with the time-bandwidth product as well. From Eq. 14 and Eq.
15 , the cross-correlation between an up-chirp and a down-chirp with the same bandwidth can also be calculated as [1]

$$
\left|R_{12}(\tau)\right|=A^{2} \sqrt{\frac{T}{B}}\left|F\left(\sqrt{B T}\left(1-\frac{|\tau|}{T}\right)\right)\right|
$$

For larger time-bandwidth products, Eq. 17 can be simplified to obtain

$$
\bar{I}_{12} \approx \sqrt{2 B T}, B T \gg 1
$$

\section{RESULTS}

Simulations have been carried out to investigate the performance of an MBR employing a linear antenna array transmitting 3 orthogonal beams (i.e. three orthogonal channels) using quasi-orthogonal linear chirps and to allow a comparison with the performance of an ESA generating the same three beams using a single waveform. The array consists of 30 ideal isotropic elements spaced of half the wavelength and simulations have been run for a carrier frequency of $15 \mathrm{GHz}$. The first channel of the MBR points at $\tilde{\theta}_{1}=5$ degrees and is generated using an up-chirp of bandwidth $B_{1}=50 \mathrm{MHz}(3 \mathrm{~m}$ range resolution) and duration $T=10 \mu \mathrm{s}$. The second channel points at $\tilde{\theta}_{2}=15$ degrees and employs an up-chirp of the same duration and bandwidth $30 \mathrm{MHz}$. Finally, the third channel points at $\tilde{\theta}_{3}=30$ degrees and is generated using a down-chirp of bandwidth $B_{3}=50 \mathrm{MHz}$ and the same duration $T=10$ $\mu$ s. The three LFM waveforms use the same frequency support and their spectra are overlapped in frequency. With no loss of generality, in this paper, we present the performance results of the MBR relative to the extraction of the first channel.

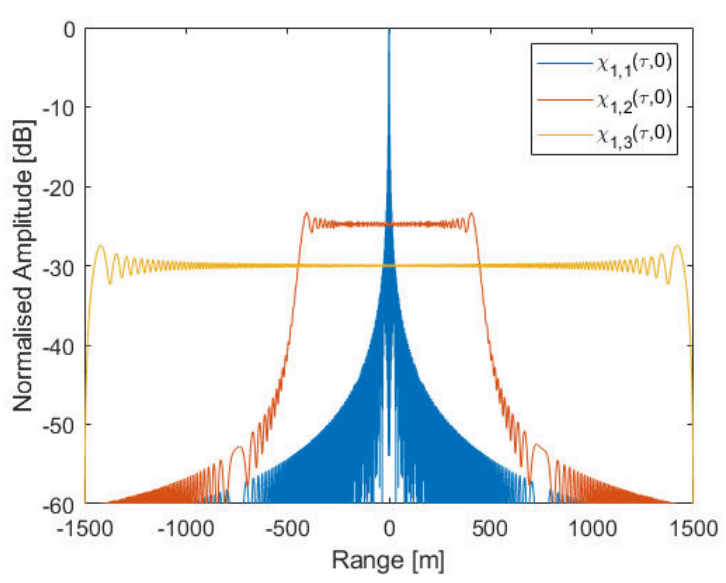

Fig. 1. Cross-correlation properties of the three waveforms employed by the MBR.

Fig. 1 shows the cross-correlation $\left|\chi_{m, k}(\tau, 0)\right|$ between the three waveforms employed by the MBR. As expected from the theoretical results, the isolation between the first and the second channels is about $25 \mathrm{~dB}$ and the isolation between the first channel and the third channel is about $30 \mathrm{~dB}$. As it is well known, the cross-correlation between channels using pseudoorthogonal LFM waveforms also presents a much wider timedomain response than that of the autocorrelation of a chirp 


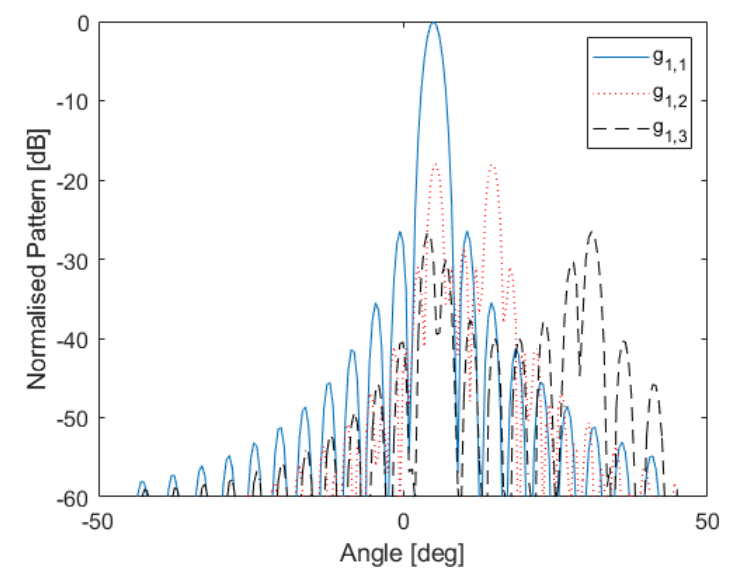

Fig. 2. Co-channel $\left(g_{1,1}\right)$ and cross-channel array pattern factors $\left(g_{1,2}\right.$ and $\left.g_{1,3}\right)$.

signal [3]. In order to characterise the array response the antenna pattern factor

$$
g_{m, k}\left(\theta_{i}\right)=\mathbf{a}^{H}\left(\tilde{\theta}_{m}\right) \mathbf{a}\left(\theta_{i}\right) \mathbf{a}^{T}\left(\theta_{i}\right) \mathbf{a}^{*}\left(\tilde{\theta}_{k}\right)
$$

is defined and analysed as a function of the target aspect angle $\theta_{i}$. The results in Fig. 2 show the co-pattern $g_{1,1}$ presents a clear peak in the direction of the co-channel at 5 degrees with sidelobes, at about $-26 \mathrm{~dB}$, that account for the sidelobe levels on transmit and on receive. The cross-patterns $g_{1,2}$ and $g_{1,3}$ present a more complex response, with multiple peaks in the vicinity of angles where the target falls in one of the three antenna beams either on transmit or on receive. It is important to stress at this point that the terms $g_{m, k}\left(\theta_{i}\right)$ are the same for both the MBR and the ESA configurations. However, for ideal MBRs, the cross-patterns are suppressed by the isolation properties between the waveforms employed.

Fig. 3 shows the normalised response of the AESA to a single ideal point target located at $3 \mathrm{~km}$ from the radar as a function of the target aspect angle. Results are relative to the first channel of the ESA, that is to the beam pointing at 5 degrees. As expected, results show a peak in the response when the target is at antenna boresight as well as a clear lower response when the target is in the transmitting beams of the other two channels. The results as a function of angle are clearly visible in the cut of the response at the target range in Fig. 4. Because the same waveform is used to generate all beams, and all cross-channel interfering contributions are such that $\chi_{k m}\left(\tau, f_{D}\right)=\chi_{m m}\left(\tau, f_{D}\right)$, the response as a function of range is that typical of a linear chirp. Fig. 6 shows the response of the 3-channel MBR. As indicated by Eq. 10, the peaks at 15 degrees and 30 degrees, relative to the target being in the cross-channel beams, are suppressed but modulated by the cross-ambiguity functions between the three waveforms. The time-domain response along the range axis is modulated by the cross-correlation functions between the chirps, presents wider sidelobes and becomes a function of the target aspect angle. The cut of the response along the angle direction at

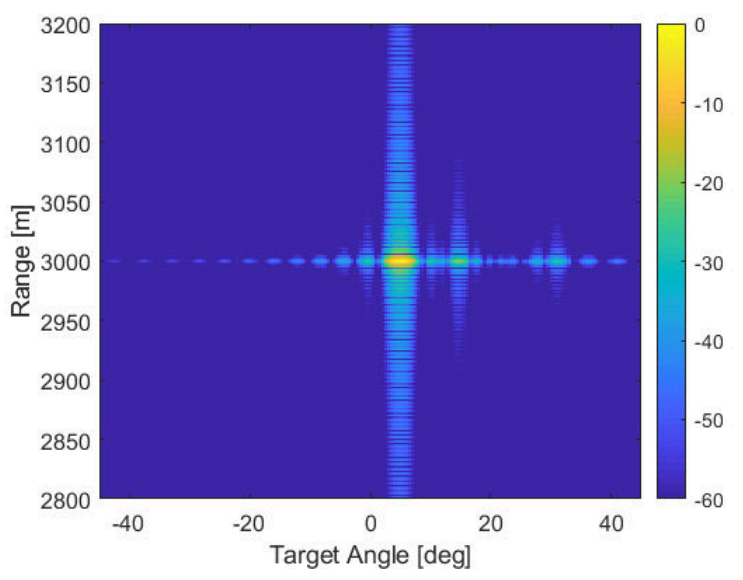

Fig. 3. Normalised response in $\mathrm{dB}$ of the single-waveform ESA configuration to an ideal point target at $3 \mathrm{~km}$ as a function of range and angle.

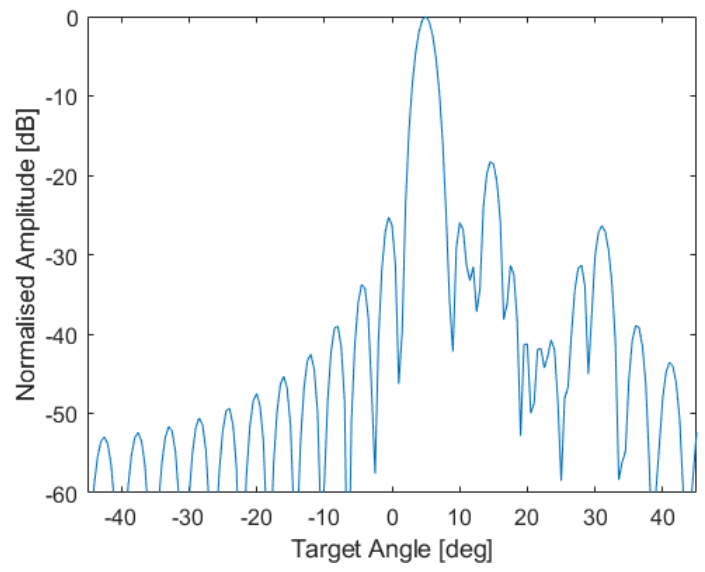

Fig. 4. Normalised response of the single-waveform ESA configuration as a function of angle at the target range.

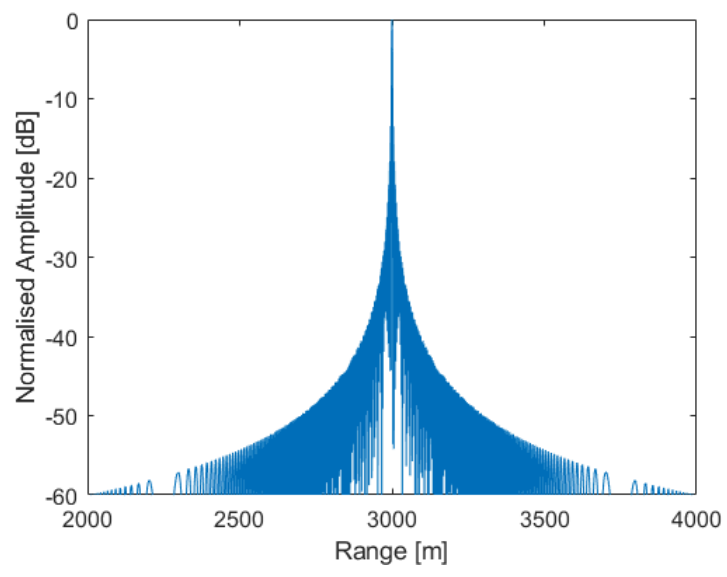

Fig. 5. Normalised response of the single-waveform ESA configuration as a function of range at the target angle.

the target range is shown in Fig. 7 and shows a much cleaner linear array response compared to that of the ESA. This is due 
to the cross-patter factors modulated by the cross-ambiguity functions being lower than the sidelobes of the co-channel array factor $g_{1,1}$. However, the range cut of the response at 5 degrees in Fig. 8 clearly shows the contribution of the crosschannel interference below the isolation levels. Swapping the waveforms employed in the second and third channels would lower the immediate cross-interference floor of about $5 \mathrm{~dB}$ but at the expense of a much longer response. Results could be clearly improved by using waveforms with increased isolation properties.

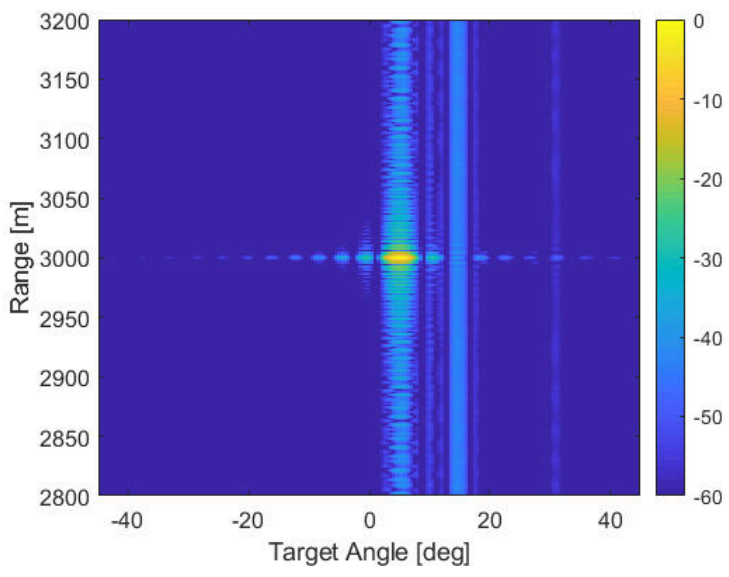

Fig. 6. Normalised response in $\mathrm{dB}$ of the 3-channel MBR to an ideal point target at $3 \mathrm{~km}$ as a function of range and angle.

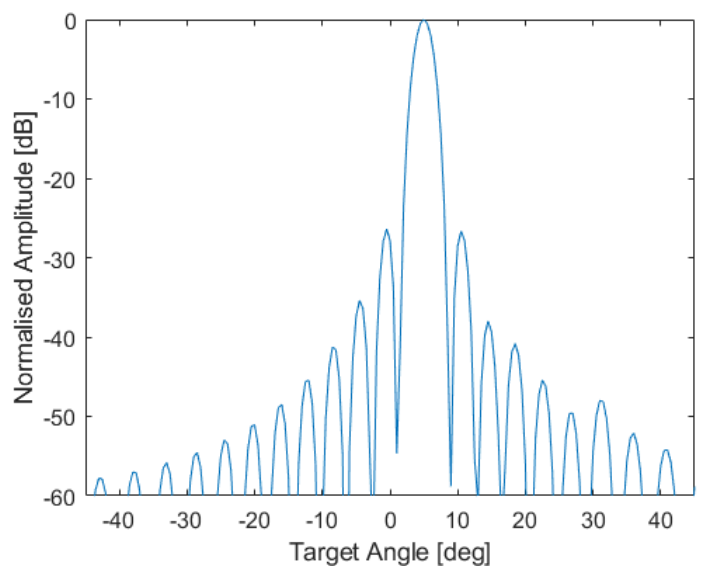

Fig. 7. Normalised response of the 3-channel MBR as a function of angle at the target range.

In order to investigate the response of MBRs to multiple targets at different distances and angles, simulations have been repeated for three targets with different Radar Cross Sections (RCS). The first two targets are in the co-channel beam (5 degrees direction) at a range of $3 \mathrm{~km}$ and $4 \mathrm{~km}$ from the radar. The target at $4 \mathrm{~km}$ presents an RCS that is $10 \mathrm{~dB}$ lower than the target at $3 \mathrm{~km}$. The third target is at $4.1 \mathrm{~km}$ with a bearing of 15 degrees but presents a much larger RCS that is $20 \mathrm{~dB}$ stronger than the target at $3 \mathrm{~km}$. Fig. 9 shows the response

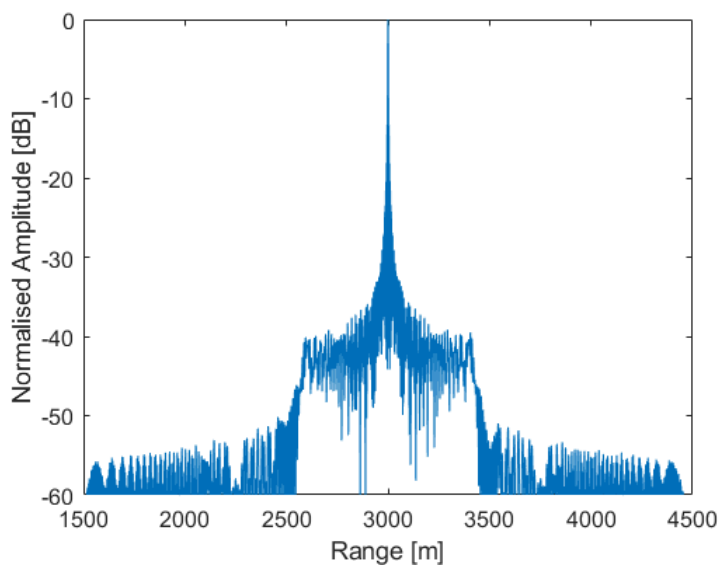

Fig. 8. Normalised response of the 3-channel MBR as a function of range at the target angle.

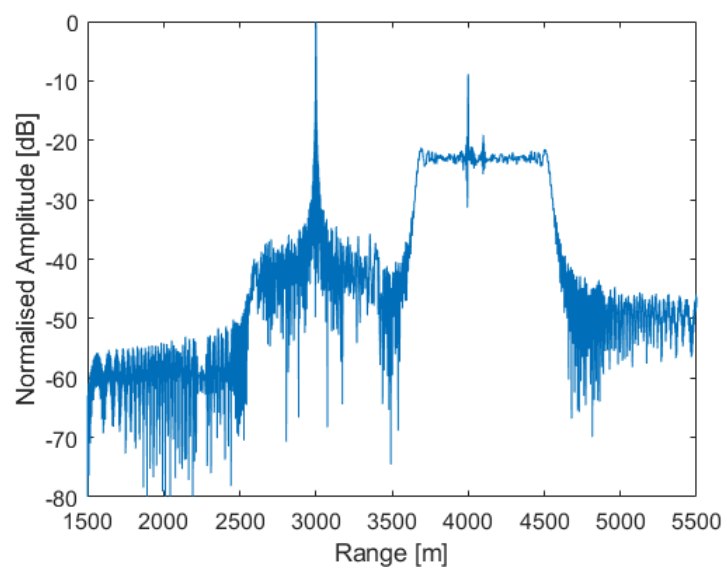

Fig. 9. MRB response to three targets with different RCS. Two targets are in the co-channel beam at a range of $3 \mathrm{~km}$ and $4 \mathrm{~km}$. The third target is located in the cross-channel beam at an angle of 15 degrees and at ange of $4.1 \mathrm{~km}$.

of the first channel of the MBR to the three targets. Results show an evident cross-channel interference centred at $4.1 \mathrm{~km}$ resulting from the presence of the strong reflector in the second channel beam. The co-channel response to the strongest target at 15 degrees, due the presence of the sidelobes of $g_{1,1}$ and that does not depend on the isolation properties of the waveforms, is also evident at $4.1 \mathrm{~km}$. The targets at 5 degrees present the highest returns and contribute with the response shown in Fig. 8, which is clearly visible for the target at $3 \mathrm{~km}$. The two targets in the co-channel remain the two strongest detections at the expense of a significant distortion of the noise floor which, for this noise-free simulation scenario, becomes dominated by the contributions of the cross-channel responses. This could be better suppressed, ideally below the thermal noise floor, with the use of better isolated waveforms. The results relative to the AESA are shown in Fig. 10. In this case, as expected, the strong target at 15 degrees is received through the antenna sidelobes and presents a higher return than the weaker target at $4 \mathrm{~km}$ in the co-channel beam. As for any ESA, the target in 
the co-channel could have been masked by the strong target at 15 degrees or its sidelobes.

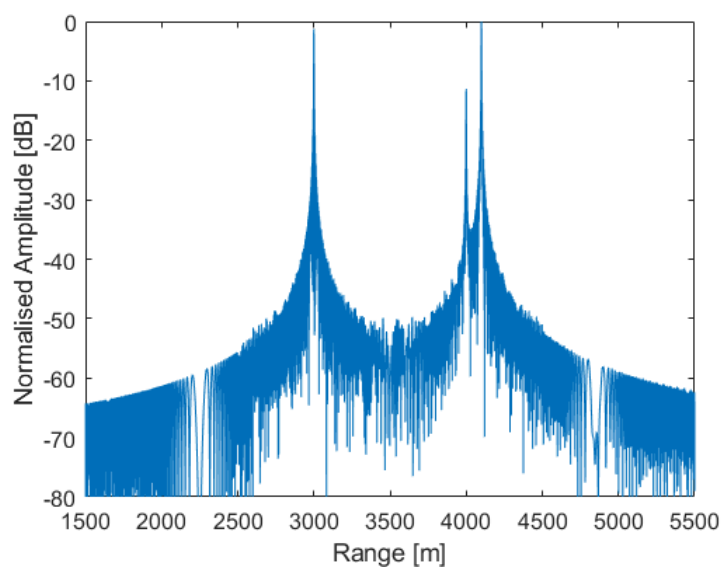

Fig. 10. ESA response to three targets with different RCS. Two targets are in the co-channel beam at a range of $3 \mathrm{~km}$ and $4 \mathrm{~km}$. The third target is located in the cross-channel beam at an angle of 15 degrees and at a range of $4.1 \mathrm{~km}$.

\section{CONCLUSiON}

In this paper, the sidelobe level properties of MBRs have been investigated. The model presented in [3] has been extended to demonstrate the analytical contribution of multiple targets to cross-channel interference and sidelobe levels. Simulation results for a 3 channel MBR exploiting quasi-orthogonal LFM waveforms have been presented to corroborate the analytical results. Results have shown that the array sidelobe levels at the target range are attenuated by the cross-ambiguity function properties between the waveforms employed. They have also shown the range response to a target, for waveforms with low isolation, suffers from cross-channel interference that can alter the noise floor characteristics of the radar.

\section{ACKNOWLEDGEMENT}

The authors would like to express their gratitude to Professor Alfonso Farina for his useful comments, to the MCM-ITP programme and Thales Optronique for funding this research.

\section{REFERENCES}

[1] G. Galati, G. Pavan, and A. De Franco, "Orthogonal waveforms for multistatic and multifunction radar," in Radar Conference (EuRAD), 2012 9th European, Amsterdam, Netherlands, 2012, pp. 310-313.

[2] L. Kocjancic, A. Balleri, and T. Merlet, "Study of the Frequency Slope Effect on the Chirp Waveform Orthogonality," in International Conference on Radar Systems (Radar 2017), no. 3. Belfast, UK: Institution of Engineering and Technology, 2017.

[3] _ , "Multibeam radar based on linear frequency modulated waveform diversity," IET Radar, Sonar \& Navigation, pp. 1-11, Aug. 2018.

[4] L. Kocjancic, A. Balleri, and T. Merlet, "Experiments of quasiorthogonal linear frequency modulated waveforms for multibeam radar," in 2019 IEEE Radar Conference (RadarConf), April 2019, pp. 1-6.

[5] J. Li and P. Stoica, "MIMO Radar with Colocated Antennas," IEEE Signal Processing Magazine, vol. 24, no. 5, pp. 106-114, Sep. 2007.

[6] J. Kantor and S. K. Davis, "Airborne GMTI Using MIMO Techniques," 2010 IEEE Radar Conference, no. 1, pp. 1344-1349, 2010.

[7] H. He, P. J. Li, and P. Stoica, Waveform Design for Active Sensing Systems: A Computational Approach. Cambridge: Cambridge University Press, Jul. 2012.
[8] F. Gini, A. D. Maio, and L. K. Patton, Waveform Design and Diversity for Advanced Radar Systems. London, UK: IET, 2012.

[9] A. Haimovich, R. Blum, and L. Cimini, "MIMO Radar with Widely Separated Antennas," IEEE Signal Processing Magazine, vol. 25, no. 1, pp. 116-129, 2008.

[10] C. E. Cook and M. Bernfeld, Radar Signals: An Introduction to Theory and Application. Boston, USA: Artech House Publishers, 1993.

[11] C. Sturm, Y. L. Sit, G. Li, H. A. Vayghan, and U. Lubbert, "Automotive Fast-Chirp MIMO Radar with Simultaneous Transmission in a DopplerMultiplex," in 2018 19th International Radar Symposium (IRS). Bonn, Germany: IEEE, Jun. 2018, pp. 1-6.

[12] H. Sun, "Performance Evaluation of Practical MIMO Radar Waveforms," 2016 IEEE Radar Conference (RadarConf), pp. 1-6, 2016.

[13] H. Li, Y. Zhao, Z. Cheng, and D. Feng, "OFDM Chirp Waveform Diversity Design With Correlation Interference Suppression for MIMO Radar," IEEE Geoscience and Remote Sensing Letters, vol. 14, no. 7, pp. 1032-1036, Jul. 2017.

[14] W.-Q. Wang, "MIMO SAR OFDM Chirp Waveform Diversity Design With Random Matrix Modulation," IEEE Transactions on Geoscience and Remote Sensing, vol. 53, no. 3, pp. 1615-1625, Mar. 2015.

[15] H. Shen and A. Papandreou-Suppappola, "Diversity and channel estimation using time-varying signals and time-frequency techniques," IEEE Transactions on Signal Processing, vol. 54, no. 9, pp. 3400-3413, 2006.

[16] Wen-Qin Wang, "Large Time-Bandwidth Product MIMO Radar Waveform Design Based on Chirp Rate Diversity," IEEE Sensors Journal, vol. 15, no. 2, pp. 1027-1034, Feb. 2015.

[17] G. Chang, A. Liu, C. Yu, Y. Ji, Y. Wang, and J. Zhang, "Orthogonal Waveform With Multiple Diversities for MIMO Radar," IEEE Sensors Journal, vol. 1748, no. 3, pp. 1-1, 2018.

[18] P. M. McCormick, S. D. Blunt, and J. G. Metcalf, "Simultaneous radar and communications emissions from a common aperture, part I: Theory," in 2017 IEEE Radar Conference (RadarConf), May 2017, pp. 16851690.

[19] P. M. McCormick, B. Ravenscroft, S. D. Blunt, A. J. Duly, and J. G. Metcalf, "Simultaneous radar and communication emissions from a common aperture, part II: Experimentation," in 2017 IEEE Radar Conference (RadarConf), May 2017, pp. 1697-1702. 